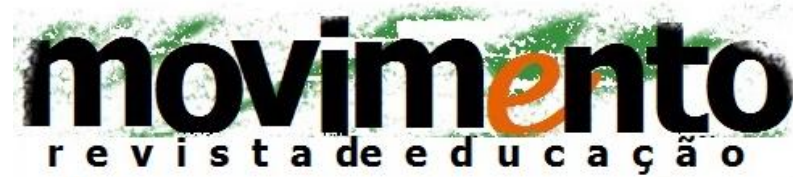

faculdade de educação - programa de pós-graduação em educação

universidade federal fluminense

issn 2359-3296

ano 3 número 4 - 2016

\section{LAS REFORMAS CURRICULARES DEL SIGLO XXI EN PERSPECTIVA HISTÓRICA}

\author{
Elisa Gavari Lisi ${ }^{1}$ \\ Título: AS REFORMAS CURRICULARES DO SÉCULO XXI NUMA PERSPETIVA \\ HISTÓRICA
}

\section{Resumo}

A primeira parte deste artigo apresenta os principais marcos do desenvolvimento da educação contemporânea. Este quadro de referência apresenta uma visão panorâmica das decisões que influíram nas reformas. $\mathrm{Na}$ segunda parte são abordados os desafios que enfrenta o curriculum na chamada terceira revolução, isto é, a sociedade do conhecimento. Aborda-se em particular a pressão exercida pelos organismos internacionais e outros intervenientes para os Estados adotarem o paradigma do lifelong-learning. Encerra-se o capítulo com algumas propostas e conclusões acerca dos limites de uma formulação educacional centrada num modelo de formação em competências.

Palavras-chave: Reformas curriculares; internalização; aprendizagem permanente

\section{Resumen}

La primera parte de este artículo presenta los hitos en la historia de la educación contemporánea. En este marco de referencia se presenta una panorámica que recoge los principios rectores de las reformas educativas. En la segunda parte se aborda los desafíos a la hora de diseñar un curriculum para la tercera revolución, esto es en el marco de las sociedades del conocimiento. En particular, se estudia la presión de los organismos internacionales a los sistemas educativos para la adopción del principio del lifelong learning o educación para la vida. Se cierra el texto con unas propuestas y conclusiones acerca de los límites de una educación centrada en la educación en competencias.

Palabras clave: Reformas curriculares; internalización; aprendizaje permanente

\footnotetext{
1 Doctora en Filosofía y Ciencias de la Educación, UNED, España; Profesora Titular de Educación Comparada adscrita al Departamento de Historia de la Educación y Educación Comparada, UNED. egavari@edu.uned.es
} 


\section{INTRODUCCIÓN}

La historia de las reformas curriculares es una parte clave de la historia de la cultura y ésta a su vez constituye un marco insoslayable para la educación de los ciudadanos. El currículo admite varias definiciones. En este artículo lo entendemos como un principio para la selección, el orden y la organización de los conocimientos para su transmisión. En este sentido es un principio de racionalización de los estudios que implica una idea previa acerca de los fines educativos en cada período y contexto de que se trate que lo enmarca y explica. Desde este enfoque podemos señalar las tendencias en la evolución del currículo en los hitos de la historia de la educación.

La primera parte de este artículo se inicia con un breve e ilustrativo recorrido que presenta los principales hitos que han marcado el devenir de la educación contemporánea. Este marco referencial ofrece una panorámica de las decisiones que han marcado las reformas en un intento por presentar el hilo conductor que ha enhebrado el devenir cultural y educativo de una parte muy importante de la historia contemporánea de Europa.

En la segunda parte se abordan los retos que afronta el currículo en la denominada tercera revolución, o mejor dicho, sociedad del conocimiento. En particular se aborda la presión desde los organismos internacionales y otras instancias para que los Estados adopten el paradigma del lifelong-learning, que tiene como eje educativo la formación en competencias. Esta tendencia se ha traducido en la adopción por parte de las universidades de todo el mundo de un currículo que traduce ciertas características de un modelo común de formación. Se cierra el capítulo con algunas propuestas y conclusiones acerca de los límites de un planteamiento educativo centrado en un modelo de formación en competencias. 


\section{LOS PRINCIPIOS QUE RIGEN EL CURRÍCULO EN LA EDUCACIÓN CONTEMPORÁNEA}

El punto de partida de la educación y cultura contemporánea se sitúa en la Revolución Francesa, que constituye un hito en la historia de la educación. En un clima de efervescencia política de la burguesía y de la necesidad del diseño de un nuevo proyecto educativo para el incipiente Estado-nación liberal se deposita la máxima confianza en la educación para el perfeccionamiento individual y social, y la consolidación de las novedosas estructuras políticas. Desde esta posición el sistema educativo en su conjunto se transformó en un puro sistema instructivo, causa y efecto del espectacular desarrollo de la ciencia y la tecnología. La pieza maestra rectora del plan de estudios era la formación intelectual, entendida como cultivo de la razón. Su primacía se basaba en el hecho de que la inteligencia estaba llamada a regir al resto de las facultades del ser humano y a orientar su conducta, buscando con ello formar personas cultivadas, autónomas y libres. Este nuevo modelo educativo implicaba un serio reajuste de la educación tradicional y la introducción de cambios esenciales en el currículo. A diferencia del modelo tradicional lego de la Edad Media, la formación religiosa se seguía recogiendo en los planes de estudio, pero muchos la relegaron a un puesto secundario o la desnaturalizaron convirtiéndola más bien en una forma de instrucción y eliminando su carácter eminentemente educativo. Algo parecido sucedió con la educación moral. Algunos tratadistas pedagógicos hablaban muy poco de ella, a pesar de sostener que era la principal faceta de la formación. Otros tenían una excesiva confianza en el poder de la razón para conducir al alumno hacia la virtud, lo que les llevaba a simplificar en exceso la educación moral.

Los ilustrados destacaron también la formación de carácter técnico y laboral. No negaban la necesidad de una formación general de carácter humanístico, sin embargo prestaron una especial atención a las habilidades más vinculadas con el trabajo productivo y el progreso económico. Asimismo proponían igualmente reducir el peso de la formación literaria, que hasta entonces había 
predominado en las escuelas, para dar cabida al espíritu científico, que era tanto o más importante. Además, había que transformar la enseñanza de las disciplinas filológicas, de manera que no se orientasen hacia la elocuencia, sino que más bien sirviesen para estimular la capacidad de raciocinio de los alumnos. Esta tensión que se inicia a partir de la Revolución Francesa entre las humanidades y las ciencias va a ser una cuestión ampliamente debatida no solamente a lo largo del siglo XIX sino también en el XX.

La Revolución Industrial, se acompañó de importantes avances en la técnica y en la ciencia, y en la industria y en las técnicas de producción. Uno de los principios fundamentales de la industria moderna es que nunca considera a los procesos de producción como definitivos o acabados, generando así el problema de la obsolescencia tecnológica en períodos cada vez más breves. Desde esta perspectiva puede afirmarse que todas las formas de producción anteriores a la industria moderna (artesanía y manufactura) fueron esencialmente conservadoras, al trasmitirse los conocimientos de generación en generación sin apenas cambios. Este cambio de enfoque también afectó al sistema educativo ya que sus fines comenzaron a ser cuestionados. La innovación y la consiguiente transformación se convierten en la esencia permanente de la modernidad y del modelo de producción. Desde estos parámetros la industria presionaba a la escuela para que produjera alumnos capaces de incorporarse a sus nuevas exigencias útiles a sus intereses productivos.

La introducción del taylorismo en Europa significó el aumento de la destreza del obrero a través de la especialización y del conocimiento técnico. Este nuevo modo de organización científica del trabajo proponía la división de las distintas tareas del proceso de producción para conseguir aumentar la productividad y la reducción de los tiempos. Al hilo de las nuevas tendencias la organización escolar pasa a ser considerada en términos de empresa (Román Pérez, 2003). Se puede aseverar que la historia de la educación del siglo XX se diferenciará de otras épocas por este enfoque en el que predomina una visión de la escuela 
cuyos objetivos curriculares se ponen al servicio de las exigencias del mundo industrial. El modelo de resultados basado en los planteamientos conductistas comienza a gobernar la planificación del currículo.

El enfoque industrial se mantiene en el mundo de la posguerra marcado por la dominación liberal en un nuevo orden político mundial (Meyer et al, 1997). En este sentido la educación de mediados de siglo se mantiene sobre las dos aspiraciones rectoras del sistema educativo. En primer lugar la lucha por el reconocimiento del derecho a la educación, inspirado en el optimismo de los ilustrados, en el nuevo marco del Estado democrático liberal caracterizado por un mayor intervencionismo en un abanico de políticas públicas. Este derecho básico se reconocería no solamente a los niños sino que debe responder a la aspiración de formar a un mayor número de personas al mayor alto nivel de formación. Se establecía la perfecta asociación entre un mayor progreso económico del Estado y el número de egresados universitarios. En segundo lugar la educación se poner al servicio de la economía y de la empresa y va adoptando cada vez más el lenguaje empresarial en todos los niveles. Las Humanidades van a ser relegadas a un cada vez más segundo plano en el currículo respecto de las materias ciencias. Estas tendencias continuarán a lo largo de todo el siglo XX y comienzos del XXI.

\section{LA UNIVERSIDAD EN LA SOCIEDAD DEL CONOCIMIENTO}

A finales del siglo $X X$ el mundo está sacudido por la tercera revolución industrial, pasando definitivamente a un modelo de sociedad centrada en los servicios o postindustrial, en la que el conocimiento se convierte en la materia prima y en el eje de la economía y del proceso de producción.

El escenario de la sociedad del conocimiento es la globalización, que es impulsada por la aceleración de la innovación tecnológica, lo que proporciona infinidad de nuevas posibilidades. Las nuevas tecnologías de la información 
permiten el auge de las redes de individuos e instituciones que llegan a reemplazar a estructuras sociales tradicionales como la Universidad (Duderstadt, 1997). En este contexto los estudios universitarios están en un proceso de revisión de sus funciones. Las tendencias en las reformas de la universidad apuntan a cambios sustanciales de sus funciones. En síntesis, se presiona a éstas para que pasen de ser universidades docentes a universidades investigadoras; y de profesionales a científicas; de pregrado a postgrado; de separadas a articuladas; de nacionales a internacionales. Se ven igualmente abocadas al cambio de metodologías de enseñanza; de la enseñanza "para saber" se pasa ahora a la "enseñanza para actuar"; de la prioridad de la función propedéutica de acceso a estudios superiores a la prioridad de la función propedéutica para la vida; del profesor propietario de la materia al coeducador; de la escuela separada a la conectada en redes medios virtuales; de la perspectiva del aprendizaje centrada en el individuo a la que incluye la interacción y el contexto.

La formación en la economía del saber se entiende como inversión en capital humano de cada sociedad, y desde este enfoque se potencia la responsabilidad de cada individuo, en tanto que sujeto del proceso de aprendizaje, que logra mantener su propia empleabilidad, capaz de asumir plenamente sus responsabilidades y compromisos, acrecentar su cultura y ejercer en plenitud todos sus derechos. Las aspiraciones de los alumnos en este marco de individualismo ya no se satisfacen con la obtención de un título para el empleo, sino que se exigen como derecho y deber poder planificar de acuerdo a sus circunstancias y posibilidades su propia educación, de tal modo que sus oportunidades de aprendizaje les permitan realizar su proyecto de vida, además de ofrecerle oportunidades culturales y de participación social (Corbella, 1997).

El discurso de las nuevas teorías de aprendizaje también insisten en considerar al individuo como núcleo al que dirigir todos los esfuerzos y al que debe formarse para desenvolverse en una sociedad compleja que requiere nuevos 
enfoques de enseñanza. El modo tradicional de currículo basado en las disciplinas de conocimiento se justificaba a través de la necesidad de transmisión de un corpus de conocimientos cerrado y acabado, y que era el necesario para la inserción laboral. En la actualidad, el trabajo de los profesionales se desarrolla en condiciones de incertidumbre, inseguridad, urgencia e impredictibilidad y el currículo debe satisfacer las nuevas necesidades que responden a una educación como un bien público internacional o un derecho de tercera generación, y una educación al servicio de la empleabilidad y de los intereses económicos.

Como ha sido la norma general a lo largo de la historia de la educación, la Educación Superior ha sido el nivel que ha recibido mayores presiones, y el primero en reformarse, provocando un efecto dominó sobre el resto de los niveles educativos. Las universidades están en un proceso de transformación como máximas representantes y productoras de conocimiento. De manera sintética podemos referirnos a que la universidad se concibe como una fase más de una cualificación, que tiene su sentido en cuanto promueve el crecimiento económico, el empleo y la productividad. Desde este enfoque, se le exige a la enseñanza superior que sea rentable, que diseñe nuevas modalidades competitivas y adopte el espíritu empresarial. Esto ha significado que en la gestión de la institución universitaria se impongan nuevos términos como "proveedores" y "clientes", "nichos de mercado", y "resultados". El marco de actuación de las universidades guiadas por los imperativos económicos no se reduce al mercado nacional sino que la universidad está llamada a tener su influencia y peso en el mercado internacional.

\section{TENDENCIAS QUE ORIENTAN EL CAMBIO EN EL CURRICULUM}

El currículo de la nueva universidad está siendo sometido a las presiones de la economía del saber. En este sentido el currículo pone un mayor énfasis en la transmisión de los saberes (renovación y adolescencia) y en las nuevas demandas que propiamente en la materia en sí y en su validez científica y 
capacidad formativa. Esta tendencia està desembocando en la pérdida del interés y preocupación por el sentido formativo y moral de los contenidos.

La misión tradicional de la universidad, que consistía en sustentar y transmitir las virtudes del conocimiento universal queda de lado y ha persistido un enfoque centrado en los procesos. Se ofrece a continuación el cambio en el énfasis entre un modelo universitario que se centra en el saber qué a otro centrado en el saber cómo:

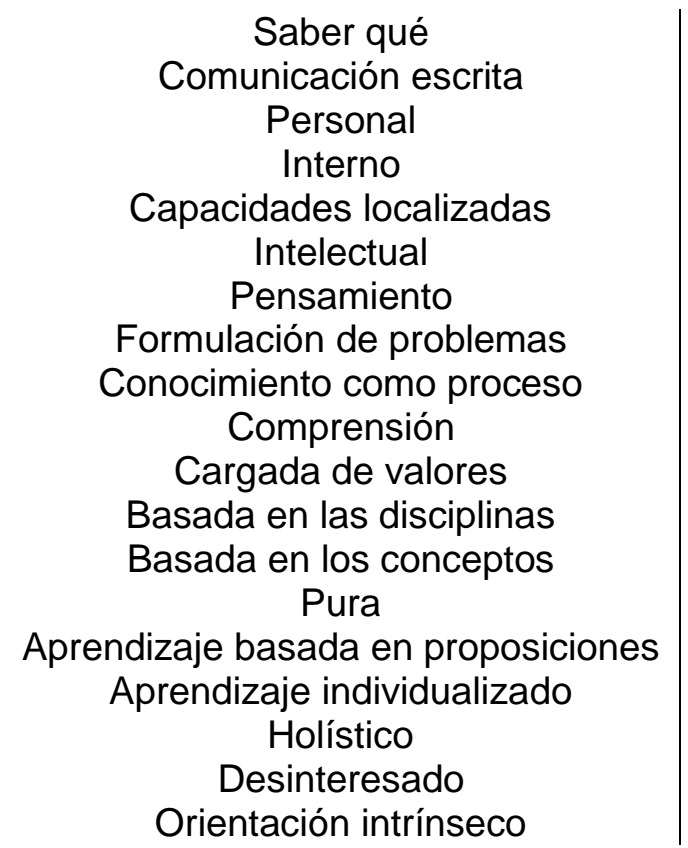

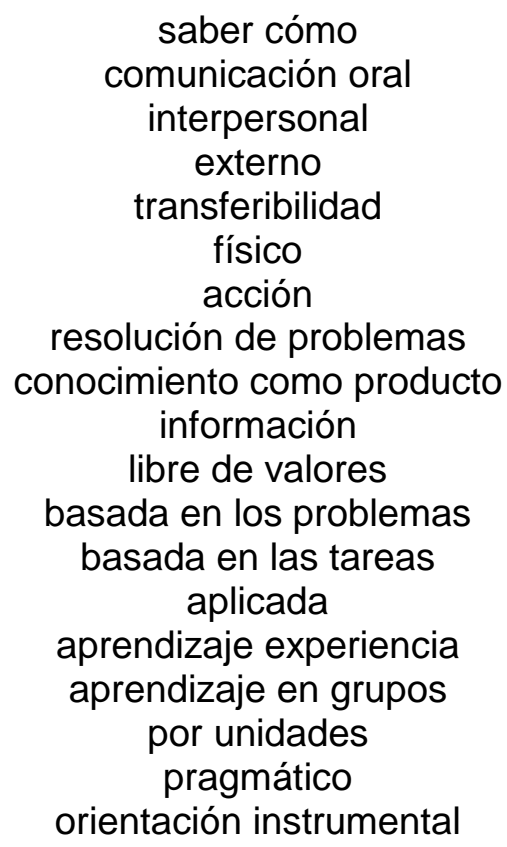

Los cambios del currículo están desafiando no solamente a los métodos de enseñanza y a las formas, procesos y contenidos curriculares, sino también a la organización institucional (contratación docente, investigación aplicada sobre investigación académica, y tutorías).

En términos generales, las universidades se han apropiado de los discursos sobre flexibilización curricular, formación basada en las competencias, sistemas modulares, enseñanza basada en la resolución de problemas, transversalidad curricular, y preeminencia del saber aplicado. A lo que se añade las tendencias que responden a la internacionalización curricular y a la 
apropiación de las nuevas tecnologías de la información y de la comunicación (ofertas académicas virtuales).

4.1. La incorporación del modelo de formación basado en las competencias en el currículo universitario

El concepto de competencia ha sido utilizado de forma continuada en el campo de la psicología, la lingüística y las teorías de la comunicación. En 1957, Chomsky define el término, refiriéndose a las capacidades y disposiciones para la interpretación y la actuación. Posteriormente este vocablo procedente de la psicología, en donde se vinculaba a la capacidad creativa, pasaría a utilizarse en el mundo empresarial, asociado a términos como eficacia y rentabilidad productiva en el área de los recursos humanos. El empleo del término competencia se remonta a 1973, cuando David MacClelland, profesor de psicología de la Universidad de Harvard, publicó el artículo, titulado Testing For Competente rather than for intelligence, que le convirtió en el padre de las competencias (Perez, 2006).

Durante los años 80 , es precisamente, cuando la terminología de competencia se extiende a nociones múltiples y diversas tales como conocimientos, capacidades, aptitudes y actitudes aunque de un modo confuso (Ortega, 2008). Los años 90 insisten en la recuperación del concepto de competencia que comienza a ganar terreno entendida como conjunto de elementos (conocimientos, destrezas, aptitudes, recursos, motivos, etc.) que están condicionadas según las condiciones de un contexto siempre cambiante y por el impacto de las nuevas tecnologías de producción y servicios (Idem).

El enfoque de competencias es heredero del conductismo que, a diferencia del modelo de rasgos, interpreta las conductas de la persona a través de la observación de sus conductas. Mientras en el modelo rasgos lo importante son las características que deben tener las personas para ocupar un determinado puesto de trabajo para el enfoque de competencias lo importante es estudiar los comportamientos observables de las personas que realizan bien su trabajo 
(eficaz y eficientemente), y, a partir, de ello, obtener criterios objetivos para desarrollar y evaluar a otras personas que deseen optar al mismo tipo de trabajo (Perez, 2006). Para este enfoque, las competencias, al contrario, que la inteligencia, la personalidad o las capacidades, no son cualidades potenciales que tiene una persona para llegar a hacer o a aprender algo, sino que son la demostración (puesta en práctica) de un saber hacer (Perez, 2006).

Las competencias constituyen el conjunto de conocimientos y de capacidades de acción adquiridas y observables, de comportamientos estructurados y modificados en función de un fin y dentro de una situación dada. Se interpreta como las características subyacentes de la persona, que están relacionadas con una correcta actuación en su puesto de trabajo y que pueden basarse en la motivación, en los rasgos de carácter, en actitudes o valores. Las características de las competencias profesionales son en síntesis tres (Barnett, 2001):

- la competencia tiene un carácter operativo y finalizado. No tiene sentido si no es con relación a la acción. Se es competente con respecto a una tarea;

- la competencia es una capacidad aprendida. No se puede negar la capacidad natural de aprender de las personas, pero la competencia se adquiere por una correlación entre teoría y práctica;

- la competencia es una noción estructurada. Combina los elementos que la componen (conocimientos teóricos prácticos, actitudes y aptitudes) para resolver un problema dentro del desempeñote las funciones del trabajador.

La sociedad moderna delega en la educación superior la tarea de desarrollar en los estudiantes las habilidades que les permitan operar de manera eficaz en la sociedad (Barnett, 2001, 32). El mismo autor insiste en que el cambio consiste esencialmente, en pasar del conocimiento como contemplación al conocimiento como operación. La ideología dominante emergente en la educación superior, es el operacionalismo que opta por dejar de lado términos como intuición, comprensión, reflexión o sabiduría y se prefieren, en cambio, otros como habilidad, competencia, resultado, información, técnica y flexibilidad (Barnett, 2001, 33). 
En la actualidad, el enfoque de las competencias se aborda desde un paradigma educativo en el que domina lo tecnológico y lo económico. Esto significa que el currículo se impone al servicio de los intereses de la economía como son: la competitividad, productividad, eficacia, eficiencia, lucro permanente, utilidades inmediatas, inversión productiva, comercialización del conocimiento y capacidad de adaptación a las situaciones cambiantes. Se han diseñado los programas de estudio, con el objeto de alentar las capacidades empresarias en todas las disciplinas y hasta en las humanidades. $Y$ en este sentido, a pesar de que se intente revestir al sistema curricular de competencias de una dimensión para la formación integral, se trata en la práctica de un sistema que busca estrechar los lazos entre la formación profesional y en las actividades laborales y productivas. Un ejemplo de ello es la definición de los contenidos curriculares a partir de las competencias laborales que se deducen de las áreas de trabajo.

\subsection{Una mayor integración entre la formación científica y la realidad}

El currículo universitario se ha visto obligado a modificar sus cimientos incorporando asignaturas como el Practicum con carácter troncal e intracurricular y cuyos fines se refieren al aprendizaje desde la experiencia. En las universidades la interrelación entre la teoría y la experiencia se materializa en los planes de estudio de cada facultad mediante la introducción de asignaturas de prácticas o a través de la incorporación de ejercicios prácticos como complemento de determinadas materias. Esta diversidad en el origen y caracterización de las prácticas permite establecer una diferenciación entre dos tipologías de prácticas: internas y externas (Pastor, 2008: 5):

Tipo de práctica

Prácticas internas

Prácticas externas (practicum)
Características

Complemento a una asignatura teórica

Trabajos prácticos

Los alumnos no se insertan en un centro de prácticas

Asignatura obligatoria contemplada en el plan de estudios (prácticas intracurriculares) 

carrera

Asignatura optativa o no obligatoria Estancias cortas o visitas al centro de prácticas Las prácticas se desarrollan en cualquier momento de la carrera

El Practicum es un proceso de aprendizaje situado en un contexto significativo en el que no hay separación entre el aprendizaje y la aplicación de lo aprendido. Es un aprendizaje en el que la cognición se sitúa en un contexto real y en el que al alumno se le obliga a afrontar diversos dilemas en los que la toma de decisiones debe basarse en el conocimiento. A partir de esa experiencia real y de los conocimientos universitarios y profesionales ya adquiridos, el alumno debe de ser capaz de llegar a la reflexión. Las virtualidades de los planes de estudio que incluyen la experiencia laboral permiten:

- motivar: conseguir que el currículo sea más significativo y tenga más sentido para los estudiantes, mejorando así sus niveles de logro académico

- investigar: permitir a los estudiantes el desarrollo de su conocimiento y comprensión del mundo laboral

- abrir horizontes: ampliar el rango de ocupaciones que los estudiantes están preparados para considerar

- ensayar: permitir a los estudiantes comprobar sus preferencias vocacionales antes de cerrarse a ellas. En ocasiones un alumno puede darse cuenta de que no les gusta intervenir en un área y prefiere formarse en otra área

- preparar: ayudar a los estudiantes a conseguir habilidades y conocimientos relacionados con un área ocupacional particular, los cuales serán capaces de aplicar si desearan entrar a un empleo en esa área

- anticipar: permitir a los estudiantes experimentar algunas tensiones del trabajo para que sean capaces de realizar su transición al trabajo de manera más confortable

- colocar: permitir a los estudiantes establecer una relación con un empleado particular que puede posteriormente contratar al alumno.

En el contexto del nuevo milenio se puede decir que el aprendizaje a través de un Practicum que sea capaz de preparar al alumno para la sociedad del conocimiento exige la adquisición de habilidades relacionadas con un aprendizaje cognitivo sofisticado, prácticas basadas en la investigación, 
formación y autoevaluación profesional continua, desarrollo y uso de la inteligencia colectiva, y con el cultivo de una profesión que valora la resolución de problemas, la asunción de riesgos, la confianza profesional, el enfrentarse al cambio y el compromiso con la mejora continua.

La normativa que se ha aprobado en este sentido es cada vez más precisa. En el caso de España se ha aprobado el Real Decreto 1393/2007, por el que se establece la regulación de las enseñanzas universitarias oficiales que se recogen en el Preámbulo con la siguiente formulación:

La posibilidad de introducir prácticas externas viene a reforzar el compromiso con la empleabilidad de los futuros graduados $y$ graduadas, enriqueciendo la formación de los estudiantes en las enseñanzas de grado, en un entorno que les proporcionará, tanto a ellos como a los responsables de la formación, un conocimiento más profundo acerca de las competencias que necesitarán en el futuro.

\subsection{El sistema modular}

En el contexto de una economía global en el que es necesario adaptarse constantemente las estructuras tienen que ser flexibles:

[...] si la Universidad es un lugar de formación para una proporción mayoritaria de la sociedad, su principal deber como servicio público será el de estar atenta a las necesidades globales de esa sociedad, que son muy distintas de las necesidades tradicionales de las élites 0 de las del propio Estado. Eso exige nuevos modelos organizativos bastante más flexibles y ágiles (Harris, 2006).

Para muchos autores (Díaz Barriga, Orozco, Díaz Villa, 2000) la denominada flexibilización alude a una categoría importada del entorno económico del modelo actual de la producción, denominado producción flexible, en contraposición al modelo fondista de producción en serie y en línea. De hecho, este tipo de propuesta se encuentra vinculada a los nuevos modelos tecnológicos. No obstante, el concepto se ha incorporado al discurso académico con mucha facilidad. La flexibilización adopta diversos sentidos en el ámbito universitario (Ramos, 2003): 
la flexibilización curricular apunta a un proceso de "apertura y redimensionamiento de la interacción entre diversas formas de conocimiento $-u$ objetos de aprendizaje- que constituyen el currículo. Se trata de adecuar el currículo a las dinámicas cambiantes tanto de los saberes como de su relación con el entorno (educación-trabajo). Se refiere también a la apertura de los límites entre los diferentes campos y área del conocimiento para fortalecer su interacción las posibilidades de integración conceptual y práctica; igualmente, a las posibilidades de diversificar las ofertas de cursos y actividades prácticas para una formación acorde con las necesidades de los estudiantes;

- la flexibilización académica referida a la generación de procesos organizativos horizontales, abiertos, dinámicos, interactivos que faciliten el tránsito de los saberes y de los sujetos sin la rigidez de las estructuras tradicionales

la flexibilización pedagógica, se refiere a la posibilidad de desarrollar diversas formas para la apropiación, socialización, reconstrucción y producción del conocimiento. La interacción enseñanza- aprendizaje puede darse en contextos diversos y con roles diferentes por parte de lo sujetos pedagógicos.

\subsection{Nuevas herramientas para evaluar el currículo}

Los cambios en el currículo llevan aparejados la utilización de nuevos instrumentos. Uno de los más innovadores es el Portafolios que se ha incorporado en todas las titulaciones para evaluar materias de carácter eminentemente práctico. El Portafolios es un instrumento de evaluación que no tiene una existencia lejana ya que, en la actualidad su uso se revela como un instrumento adecuado para la formación de profesionales reflexivos en el marco de la educación a lo largo de la vida. El uso de los Portafolios para la formación de profesionales de la educación se generalizó en los EEUU en el decenio de los 80. La crisis de la reforma educativa de los setenta y la insatisfacción generalizada respecto al rendimiento académico de los alumnos alertaron acerca del bajo nivel educativo de los EEUU. Así lo señalaban informes tales como el Nation at risk (Una nación en riesgo), que criticaban la educación que en esos momentos se impartía en las aulas de dicho país. A finales de la década de 1980, los responsables de la nueva reforma educativa consideraron que era necesario introducir conceptos nuevos en la educación norteamericana, que permitieran la aplicación inmediata de la enseñanza de habilidades intelectuales superiores y el desarrollo de la capacidad de los estudiantes para ejercer el juicio personal (Lyons, 1999, 15). 
La palabra Portafolios tiene distintas acepciones. La primera se refiere a la "cartera que se usa para llevar documentos públicos"; esta acepción se refiere al cargo y las funciones de un ministro (Websters, 1963: 662). En este sentido se comprende como una credencial. La segunda acepción nos define el Portafolios como un "maletín para guardar, generalmente sin doblar, hojas sueltas de papel, impresos, etc" (ibidem: 662). En un tercer sentido, el Portafolios sería el resultado de un proceso dinámico mediante el cual los profesionales de la educación reúnen los datos acerca de su trabajo y crecimiento profesional, agrupados y redactados por ellos con cuidadosa reflexión, y que nos permiten comprender el sentido de sus actuaciones educativas.

La tercera acepción es la que conviene al Portafolios como instrumento de evaluación del alumno en el Practicum. En este sentido nos permite analizar al profesional de la educación que está formado no de manera genérica, sino en una realidad sobre la que interviene y sobre la que posteriormente reflexiona. El Portafolios recoge toda una serie de documentos que sustentan una forma personal de intervención: "Es una especie de espejo que refleja el crecimiento profesional, así como la competencia y eficacia profesional del que lo elabora”. Y lo mismo que un espejo uno se mira, ve lo que le gusta y lo que no, y lo puede cambiar. Y después volverse a mirar y cambiar más cosas. Incluso dejar que lo miren otros e incluir nuevos cambios.

\section{INTERNACIONALIZACIÓN DEL CURRÍCULO}

En un contexto en el que se está llevando a cabo una mayor integración económica internacional, acompañada de la firma de acuerdos regionales y globales de comercio e inversión, y de las posibilidades que brindan las tecnologías de la información, el sistema educativo está más que nunca abierto a la cooperación internacional. El currículo se adapta a esta internacionalización adoptando nuevas modalidades (Ramos, 2003): aplicación de un enfoque internacional comparativo en los diferentes análisis; inclusión de 
ofertas que preparan a los estudiantes para profesionales internacionales definidas (manejo de estudios internacionales, contabilidad); estudios en lenguas o de lenguas extranjeras, que específicamente se aboquen a temas de comunicación y que proporcionen capacitación y habilidades interculturales; estudios que forman parte de programas interdisciplinarios tales como un estudios de un área o región geográfica; reconocimiento internacional de títulos profesionales.

Asimismo las universidades tienden a que sus estructuras sean similares y que los grados académicos tengan reconocimiento internacional. Desde este enfoque el currículo tiene que permitir el intercambio de servicios educativos y se adapta a las siguiente modalidades (Idem): programa de estudios operados fuera del país oferente (como estudios en administración de empresas); campus de una universidad o centro de estudios establecida en un país extranjero; operaciones de franquicia; arreglos de universidades hermanas, en donde un título se cursa en más de una universidad; educación a distancia, ya sea por medio electrónico u otros; estudios en el exterior; ventas de materiales propios, como libros o exámenes, además de los servicios educativos.

Otro nuevo sello del modelo educativo del siglo XXI es la tendencia a incorporar parte del currículo en inglés, que se ha convertido en el idioma del nuevo milenio, ya que este idioma aspira a desempeñar el papel de lingua franca ejercido por el latín en tiempos pretéritos. En la actualidad el inglés es el idioma de las revistas consideradas de impacto internacional, que se califican en las bases de datos que también están recogidas en este idioma. En este sentido las universidades y académicos de los docentes de habla inglesa gozan de ciertas ventajas respecto de otros. El sistema de revisión de pares está dominado por personas acostumbradas al lenguaje y metodología de los académicos norteamericanos. Este dominio científico del inglés también se refleja en los materiales académicos en lengua inglesa que predominan en el mercado. Se puede decir que la internacionalización del currículo responde a una tendencia clara el dominio desde las universidades del Norte sobre las del 
Sur. Por último, cabe decir que en algunos estudios se vislumbra una fuerte preponderancia del currículo en inglés. Éste es el caso del sector empresarial o de gestión de los negocios.

\section{El caso de Europa: el Proceso de Bolonia}

En los comienzos del nuevo milenio la política educativa europea se ha encaminado a la consecución de un nuevo objetivo estratégico, como es, que Europa se convierta para el año 2010 en la economía basada en el conocimiento más competitiva y dinámica del mundo, capaz de crecer económicamente de manera sostenible con más y mejores empleos y con mayor cohesión social. Para la consecución de este fin los sistemas educativos europeos están en un proceso de convergencia. En el nivel de Educación Superior destaca la creación del Espacio Europeo de Educación Superior y en el nivel escolar se han establecido unos objetivos comunes.

La construcción del EEES tiene sus raíces en el Tratado de Maastricht (1992). De un modo más específico, este proceso se inicia con la Declaración de la Sorbona de 1998, que destacó el papel de las Universidades en el desarrollo de la dimensión cultural europea y de la Europa del conocimiento, y se consolida y se amplía con las Declaraciones de Bolonia (Junio de 1999), de Praga (2001) y de Berlín (Septiembre de 2003) y Bergen (Mayo de 2005). En ellas los Ministros europeos acuerdan promover y desarrollar en sus respectivos países la reforma de la estructura y la organización de las enseñanzas universitarias para estimular la construcción de un espacio europeo de educación superior. Actualmente, este proceso de convergencia, conocido como Proceso de Bolonia, es apoyado por más de 4000 Universidades y Centros de Educación Superior europeos, de países de más de 40 naciones, que incluyen desde Alemania, Francia hasta Rumania, Eslovaquia, Grecia, Turquía, Estonia o Lituania. 
Desde la Declaración de Bolonia las funciones que se asignan a la Universidad son las siguientes:

- la capacitación profesional de los universitarios en los valores que demandan los puestos de trabajo de la Sociedad del conocimiento;

- la promoción de un espacio europeo de aprendizaje permanente;

- la formación para el ocio y para la ocupación racional del tiempo libre, como modo de autorrealización personal.

La promoción de un espacio europeo de aprendizaje permanente se reconoce explícitamente en la Declaración de Praga de 2001, que considera función de la Universidad: "el aprendizaje a lo largo de la vida, como elemento esencial para alcanzar una mayor competitividad europea, mejorar la cohesión social, la igualdad de oportunidades y la calidad de vida"; la necesidad, en suma, de "hacer realidad un espacio europeo de aprendizaje permanente" (Comisión Europea, 2001).

En una Sociedad del conocimiento la formación no debe considerarse como un proceso efímero, asociado a una etapa de la vida, sino como un ciclo que se extiende a toda la vida activa de la persona. Esa sociedad reclama de su capital humano una adaptación continua del conocimiento que reduzca la distancia entre desarrollo científico y sus aplicaciones sociales, a la par que desarrollen, de modo permanente, la capacidad de razonamiento y de integración de cualquier información innovadora. A esto se añade que en los textos comunitarios se pretende que, a través del aprendizaje permanente a lo largo de la vida no solamente se consiga alcanzar una mayor competitividad europea, sino también mejorar la cohesión social, la igualdad de oportunidades y la calidad de vida.

\subsection{Currículo que se define por las competencias}

En la actualidad, y en particular, desde el Consejo de Lisboa en el año 2001, en un contexto económico marcado por la necesidad de mejorar la empleabilidad y adaptabilidad de los ciudadanos ante los elevados niveles de desempleo 
estructural, se apuesta por ofrecer una enseñanza de calidad. Desde las instituciones comunitarias la mejora de la calidad educativa se tradujo en la revalorización del concepto de aprendizaje permanente como base de las políticas y acciones educativas concretas europeas. Los documentos comunitarios comenzaron a utilizar esta idea bajo los términos de la educación y formación a lo largo de la vida o la expresión anglosajona lifelong learning, especialmente a partir de la proclamación de 1996 como año del aprendizaje permanente, momento en el que el debate acerca de la importancia de este concepto se intensificó. Finalmente, el Tratado de Amsterdam, estableció formalmente que el aprendizaje permanente se erigía como principio rector de la política comunitaria en el campo de la educación y la formación europea.

La educación permanente como estrategia implica cambios radicales en el pensamiento educativo convencional, en los métodos, organizaciones, estructuras y prácticas. En este marco de reforma el elemento central al concepto de aprendizaje permanente es que todas las personas aprenden en todas las etapas de su existencia, por lo que todos los niveles educativos son capaces de contribuir a tal fin. Esto supone una revisión en profundidad del nivel escolar y del universitario.

La escuela deja de ser esencialmente el lugar en donde se produce el proceso de adquisición de los conocimientos y se convierte simplemente en el preludio del aprendizaje. El rol de la escuela cambia y ya no se espera de ella que ofrezca el aprendizaje de diversas materias sino que debe educar a los estudiantes en la capacidad de aprender a aprender. El currículo escolar no debe limitarse a la retención y repetición de los conocimientos propuestos, sino que se centra en el desarrollo de las competencias consideradas básicas. Otro de los cambios que se introducen en el currículo se refieren a que la finalidad del aprendizaje permanente es el desarrollo de la personalidad de cada individuo, de su originalidad. El currículo debe permitir el desarrollo de cada individualidad humana. La escuela está basada en patrones fijos de años, de contenidos, de metodologías, etc, y pensada para individuos de igual 
capacidad y ritmo de aprendizaje. El currículo en un sistema de aprendizaje permanente debía permitir adquirir al alumno los conocimientos, habilidades, actitudes, valores y la auto-imagen necesaria para seguir aprendiendo en el futuro, es decir, los prerrequisitos personales para el aprendizaje.

El papel de la universidad es fundamental en el sistema educativo y en la adopción del aprendizaje permanente. Una de las transformaciones más importantes que ha introducido la adopción de este principio ha sido la extensión de la educación de este nivel a un público nuevo y más amplio. Una de las estrategias más utilizadas para este fin ha sido la utilización de métodos de enseñanza a distancia y del uso de las nuevas tecnologías, lo que ha introducido una gran flexibilidad en el sistema y en el proceso de aprendizaje. Hasta ahora el estudiante culminaba sus estudios con la graduación. Ahora el fin de los estudios no es más que el comienzo de una vida de aprendizaje. No obstante la universidad debe organizar sus programas de formación de manera que los estudiantes adquieran la capacidad y la motivación para continuar aprendiendo durante toda su vida, no sólo en los contextos formales, sino también en el trabajo o en la comunidad. La universidad debe esforzarse en crear ambientes y experiencias que lleven a los estudiantes a descubrir y construir el conocimiento por ellos mismos, para convertir a los estudiantes en miembros de comunidades de aprendices que hacen descubrimientos y resuelven problemas. Por último, mencionar la importancia de que en las certificaciones se recojan las competencias del alumno universitario.

El proyecto Tunning (2003) está sirviendo de referencia para muchas universidades, y propone el sistema de competencias como lenguaje común para describir los objetivos de los títulos y planes de estudio, así como referencia para la evaluación de los resultados de los alumnos. En concreto, la Agencia Nacional de Evaluación de la Calidad y Acreditación (ANECA) ha tomado como base de referencia la protesta Tunning para la formulación de las competencias transversales de las nuevas titulaciones en las universidades españolas. El Proyecto Tunning ha llegado a la propuesta de un conjunto 
determinado de competencias generales que deben guiar la reforma educativa. De las 85 competencias iniciales se eligieron las 30 más valoradas tanto por académicos, como empleadores y graduados:

\section{COMPETENCIAS PROYECTO TUNNING}

\begin{tabular}{|c|c|c|}
\hline $\begin{array}{l}\text { Competencias } \\
\text { instrumentales }\end{array}$ & $\begin{array}{l}\text { Capacidad de análisis y } \\
\text { síntesis } \\
\text { capacidad de organizar y } \\
\text { planificar } \\
\text { conocimientos generales } \\
\text { básicos } \\
\text { conocimientos básicos de } \\
\text { la profesión }\end{array}$ & $\begin{array}{l}\text { conocimiento de una } \\
\text { segunda lengua } \\
\text { habilidades básicas en el } \\
\text { manejo de ordenadores } \\
\text { habilidades de gestión } \\
\text { información } \\
\text { resolución de problemas } \\
\text { toma de decisiones }\end{array}$ \\
\hline $\begin{array}{l}\text { Competencias } \\
\text { interpersonales }\end{array}$ & $\begin{array}{c}\text { capacidad crítica y } \\
\text { autocrítica } \\
\text { trabajo en equipo } \\
\text { habilidades } \\
\text { interpersonales } \\
\text { capacidad de trabajar en } \\
\text { un equipo interdisciplinar } \\
\text { capacidad para } \\
\text { comunicarse con expertos } \\
\text { de otras áreas }\end{array}$ & $\begin{array}{c}\text { apreciación de la } \\
\text { diversidad y } \\
\text { multiculturalidad } \\
\text { habilidad de trabajar en un } \\
\text { contexto internacional } \\
\text { compromiso ético }\end{array}$ \\
\hline Competencias sistémicas & $\begin{array}{c}\text { capacidad de aplicar los } \\
\text { conocimientos en la } \\
\text { práctica } \\
\text { habilidades de } \\
\text { investigación } \\
\text { capacidad de aprender } \\
\text { capacidad para adaptarse } \\
\text { a nuevas situaciones } \\
\text { capacidad para generar } \\
\text { nuevas ideas (creatividad) }\end{array}$ & $\begin{array}{c}\text { liderazgo } \\
\text { conocimiento de culturas } \\
\text { y costumbres de otros } \\
\text { pueblos } \\
\text { habilidad para trabajar de } \\
\text { forma autónoma } \\
\text { diseño y gestión de } \\
\text { proyectos } \\
\text { iniciativa y espíritu } \\
\text { emprendedor } \\
\text { preocupación por la } \\
\text { calidad } \\
\text { motivación de logro }\end{array}$ \\
\hline
\end{tabular}

Fuente: Competencias del Proyecto Tuning (Gonzalez y Wagenaar, 2003) 
Las competencias no se van a introducir solamente en la universidad sino también en los restantes niveles educativos. A partir del Consejo de Ministros de Lisboa se acordó el informe Los objetivos futuros concretos de los sistemas de educación. En este documento se identifican tres objetivos específicos que deben incorporar los sistemas educativos en los próximos diez años. La soberanía acerca de los contenidos permanece en manos de los distintos Estados, pero es aconsejable que estén en consonancia con la Recomendación del Parlamento y el Consejo Europeo de 2006 sobre las competencias clave para el aprendizaje permanente. En dicho documento se afirma que "las competencias se definen como una combinación de conocimientos, capacidades y actitudes adecuadas al contexto. Las competencias clave son aquéllas que todas las personas precisan para su realización y desarrollo personales, así como para la ciudadanía activa, la inclusión social y el empleo. "Dichas competencias deberían desarrollarse a lo largo de la enseñanza obligatoria y actualizarse en el aprendizaje a lo largo de la vida. El marco de referencia establece ocho competencias clave:

1. comunicación en lengua materna

2. comunicación en lenguas extranjeras

3. competencia matemática y competencias en ciencia y tecnología

4. competencia digital

5. aprender a aprender

6. competencias sociales y cívicas

7. sentido de la iniciativa y espíritu de empresa, y

8. conciencia y expresión culturales.

6.2. La flexibilización del currículo y la introducción del ECTS como unidad de valor

La construcción del EEES de aprendizaje permanente requiere transformaciones significativas en los sistemas de educación europeos y la introducción de tres elementos: una nueva estructura de títulos en dos niveles (grado y postgrado), una nueva forma de acreditar las competencias adquiridas por los alumnos en su proceso formativo (suplemento europeo al título) y el crédito ECTS o crédito europeo. La adopción del crédito europeo hará posible y facilitará el reconocimiento académico completo y "a priori" de los estudios 
realizados en cualquier país de la Unión Europea; la movilidad estudiantil; el desarrollo de un currículo homologable internacionalmente; y la transferencia y comparabilidad de los aprendizajes de una institución a otra.

La adopción del ECTS supone un sistema centrado en el aprendizaje del alumno que se fundamenta en la cantidad de trabajo necesaria para que el alumno consiga sus objetivos de un programa, objetivos que se expresan en términos de resultados de aprendizaje y competencias que deben de adquirirse (Karseth, 2006, 266):

\begin{tabular}{|c|c|c|c|}
\hline Estructura & Contenido & Pedagogía & Objetivos \\
\hline Módulos & Conocimiento & Enseñanza centrada en el & Objetivos centrados \\
\hline & $\begin{array}{l}\text { Mercado como } \\
\text { referencia }\end{array}$ & $\begin{array}{l}\text { Relaciones de proveedor- } \\
\text { consumidor }\end{array}$ & $\begin{array}{c}\text { Habilidades } \\
\text { transferibles/genéric } \\
\text { as }\end{array}$ \\
\hline
\end{tabular}

\section{CONTRADICCIONES DE UN CURRÍCULO CENTRADO EN UNA FORMACIÓN BASADA EN LAS COMPETENCIAS EN EL MARCO EUROPEO}

Analicemos el caso de Europa; las políticas educativas europeas han sido ciertamente tímidas en comparación con las económicas o políticas. Desde hace tiempo manejamos el euro y el pasaporte europeo y, sin embargo, solamente a partir del año 2010 podemos hablar del Espacio Europeo de Educación Superior. Desde el año 2001, la política educativa se ha puesto al servicio de la economía, en el marco del aprendizaje permanente. En este nuevo escenario, el concepto de aprendizaje permanente se ha convertido en el paradigma rector de las políticas educativas para preparar a los ciudadanos para la sociedad del conocimiento y está influyendo, en gran medida, en las políticas públicas educativas de los distintos Estados. Esta política educativa pretende la consecución de un Espacio Europeo de Educación para una Europa de los ciudadanos inmersos en una sociedad del conocimiento, lo que supone un doble objetivo: la empleabilidad de los mismos desde un enfoque 
economicista y de acuerdo al principio de la competitividad; y la integración y cohesión social desde un enfoque de Europa de los ciudadanos y de acuerdo al principio de la igualdad de oportunidades. Esta duplicidad de objetivos resulta ciertamente contradictoria.

En cuanto a las dificultades para llegar a un acuerdo acerca del contenido común concreto que debe enseñarse a los europeos, lo único que cabe señalar es que se ha conseguido establecer un mínimo común: las competencias. Los ocho dominios de las competencias clave que establecen las instituciones comunitarias (Comisión Europea, 2004) son las siguientes: comunicación en la lengua materna; comunicación en una lengua extranjera; competencia matemática y competencias básicas en ciencia y tecnología; competencia digital; aprender a aprender; competencias interpersonales y sociales; espíritu emprendedor; y, por último, expresión cultural. La mayoría de estos ámbitos de competencias se refieren a aspectos vinculados a una educación que forme para la empleabilidad y la adaptabilidad. Ante esta propuesta podemos presentar una contrapropuesta que prepare a ciudadanos en su dimensión personal y social, formando a sujetos autónomos que sean auténticos ciudadanos y que contribuya a la sostenibilidad de la sociedad.

A partir de la Conferencia de Berlín de 2003 la Unión Europea intenta vincular la construcción del Espacio Europeo de Educación Superior con el mundo de los valores, la cohesión social, la reducción de las desigualdades sociales y de género. En este sentido venimos a considerar que la educación para la formación de ciudadanos comprometidos con la inclusión social y la sostenibilidad debería incluir la formación en dos tipos de competencias: genéricas y éticas (Martínez, y Esteban, 2005). Las genéricas pueden desarrollarse mediante una materia concreta, mientras que las éticas se desarrollan ejercitándolas en muchas materias y en contextos de aprendizaje diversos. Las primeras suponen articular coherentemente un conjunto de conocimientos, aptitudes, valores y actitudes (Rye y Torbjorrsen, 2004), y movilizarlos para aplicarlos en el ejercicio cotidianos de la ciudadanía. Las 
segundas se refieren a un conjunto semejante de saberes, sentimientos y movilizarlos para construir un modelo de vida personal, elaborado de manera autónoma y en situación de interacción social, que goce de las características necesarias para ser un "modelo de vida buena" y, a la vez un "modelo de vida sostenible".

El aprendizaje en competencias éticas es especialmente relevante para la inclusión social y la sostenibilidad. La acción pedagógica se articularía en torno a tres objetivos: que la persona sea capaz de construir y guiar con criterio su modo y estilo de vida buena; que la persona aprenda y acepte como valioso sólo aquellos modos y estilos de vida que se sustentan en criterios de justicia, equidad y dignidad; que la persona aprenda el ejercicio de derechos y deberes ciudadanos y desarrollo la participación eficaz en contextos de debate. Y sobre todo que no se deje de lado el espíritu de solidaridad

Algunos filósofos de la educación nos proponen alternativas que no se centran exclusivamente en planteamientos curriculares basados en las competencias y en los resultados a obtener, sino que se dirijan a la consecución de una educación integral de la persona. Las orientaciones a potenciar que nos proponen estos autores se dirigen al desarrollo de tres dimensiones fundamentales de la persona, como son, la afectividad, imaginación y solidaridad (Altarejos, Rodríguez Sedano y Fontrodona, 2003: 143,167,190). Se sintetizan en las siguientes propuestas:

a) desmitificar la exagerada importancia educativa atribuida al auto concepto, pues remite peligrosamente a la mismidad objetivada y magnifica los sentimientos de seguridad: hay que aprender a afrontar el riesgo ya a admitir deportivamente el fracaso como un elemento normal de la vida;

b) abandonar las secuelas pedagógicas- que aún son muchas y fuertes- del mastery learning: más que el dominio de lo aprendido se debe propiciar el dominio de uno mismo desde la libertad; tanto como la formación intelectual debe cuidarse la formación del carácter y la educación de la afectividad;

c) potenciar la confianza del educador en sus acciones, enseñanzas y sobre todo en las personas que educa; no temer al engaño, a los posibles traumas y a los probables abusos de los educandos: hacer del sentido del humor un faro orientador de la enseñanza; 
d) rehabilitar la educación estética que forma la sensibilidad, condición primaria de la formación humana: asistir al desarrollo de las capacidades sensoriales dedicándose al cuidado esmerado de sus múltiples manifestaciones, desde la higiene corporal hasta el gusto gastronómico;

e) promover la moderación como actitud básica en el uso de las cosas materiales, en las actividades lúdicas y en el trato humano con los iguales, superiores e inferiores: quien no sabe habérselas y tratar con el entorno se vacía y se pierde a sí mismo en la exterioridad, tantas veces alienante;

f) rechazar el supuesto valor de la competitividad, que sólo lo es para el pensamiento liberal-individualista, remplazándolo por la emulación, que lleva a la superación de uno mismo inseparablemente de la mejora del émulo;

g) fomentar las tareas cooperativas en el aprendizaje, lo que es distinto en buen parte del aprendizaje cooperativo: no aprenden todos, sino cada uno, pero para ser plenamente uno se debe aprender con otros

h) potenciar el aprendizaje en la acción, lo que tampoco supone reavivar la vigencia del principio de actividad en la educación, sino referir la enseñanza valor formativo de las narraciones;

i) resaltar el valor de la belleza, que congrega por sí misma sensibilidades diversas; donde el encuentro interpersonal e intercultural se hace fácil, viable y fecundo es en la comunicación estética, mucho más que en el diálogo cultural o el consenso ético;

j) acrecentar el estudio de las disciplinas humanísticas no sólo equiparándolas, sino incluso superando a las científicas en el diseño y desarrollo curricular; y más todavía; rescatando el hondo cometido y el latente sentido humanístico que tiene la ciencia, hoy bastante deprimido por las teorías formales y los resultados de la investigación;

k) promover la formación de hábitos sociales y solidarios que desarrollen las tendencias naturales y latentes en la sociabilidad humana y en la dimensión personal de la coexistencia.

\section{CONCLUSIONES}

El modelo curricular que se propone en la actualidad responde no tanto a un modelo educativo sino a otro basado en una formación centrada en las competencias. Este modelo no prima el valor del contenido de la materia sobre los procesos, sino que da mucha importancia a las exigencias del contexto en el que se formule el modelo educativo. Desde esta perspectiva, como señala Bastien (1997, 32), un experto es competente porque: a) domina muy rápido y con seguridad las situaciones más corrientes, puesto que dispone de esquemas complejos que pueden entrar inmediatamente en acción; b) es 
capaz, a través de la reflexión, de coordinar y diferenciar rápidamente sus esquemas de acción y sus conocimientos para enfrentar situaciones nuevas. Esto supone el traslado del experto en la materia a uno centrado en los procesos. Asimismo el diseño de los contenidos de formación se basan más en la inserción laboral, que en el desarrollo de conocimiento por la validez del aprendizaje del mismo. Esto es así porque a partir de los años setenta, y en particular en la década de los ochenta, el modelo de competencias se ha trasladado desde el ámbito de los recursos humanos en la empresa al sistema educativo. La investigación en este campo aportaba evidencias sobre el papel de las variables no cognitivas y el desempeño laboral, pero ciertamente las virtudes del modelo han sido sobrevaloradas. A partir del Consejo de Ministros europeos celebrado en 2001 y a través del Plan de Bolonia, cuyos impulsores son políticos y administradores, y no los mismos académicos, este paradigma se ha convertido en el rector de las políticas europeas de educación y formación profesional. Posiblemente el éxito de su aplicación en empresas líderes haya sido uno de los factores a tener en cuenta a la hora de incorporar el modelo de formación en empresas como núcleo de la política educativa europea. La idea-fuerza que subyace consiste en aumentar la empleabilidad de los ciudadanos comunitarios para conseguir convertir a Europa en el área de conocimiento más competitiva del mundo, frente a otras potencias mundiales como los Estados Unidos. Y este objetivo nada tiene que ver con la formación integral de la persona como ha sido el objetivo de la pedagogía en una notable parte de la historia de la educación.

El enfoque de una educación basada en formación a través de las competencias ha sido muy criticado. Las competencias tienen aspectos positivos como su capacidad integradora; su transferibilidad; multifuncionalidad, y carácter dinámico, y su susceptibilidad de evaluación. Clasificadas en generales, específicas o particulares, según el campo de aplicación se ha subrayado su importancia para el logro de las finalidades educativas a lo largo de la vida. De hecho la planificación curricular a través de las competencias se ha generalizado tanto en las enseñanzas básicas, como en el nivel superior, y 
en la educación permanente para toda la vida. Esto no está exento de dificultades. En ocasiones, se está produciendo un fenómeno de moda en el más superficial de los sentidos, de modo que se adopta la nueva terminología pero no se asumen o no se aplican los principios básicos en profundidad. Incluso en ocasiones se produce una confusión terminológica y conceptos como habilidades, actitudes 0 rasgos son utilizados como etiquetas intercambiables. En otras ocasiones el concepto de competencia se convierte en un cajón de sastre en el que todo tiene cabida (comportamientos, rasgos, cogniciones, actitudes, conocimientos, motivaciones...).

A esto se añade que en el marco de la sociedad del conocimiento las competencias se apropian de conceptos nuevos como la participación activa del estudiante, la determinación de los contenidos curriculares a partir del quehacer de los expertos en los lugares de trabajo, se privilegia el uso del conocimiento frente a la producción del conocimiento, la alternancia (aulas académicas y lugares de trabajo), la formación polivalente y la capacidad de transferencia de las habilidades y destrezas adquiridas de un contexto a otro. Esto no está exento de riesgos ya que es un modelo fundamentalmente dependiente de los intereses de los agentes externos al sistema educativo impidiéndose así que sean los miembros de la academia, expertos en las materias, quienes decidan cómo organizar éstas desde los parámetros que deben de guiar la investigación, la producción, la difusión y sobre todo la docencia y su aplicación curricular. En este sentido, la universidad ha pasado de ser un espacio privilegiado de conocimiento con autonomía propia a constituirse en un eslabón más de la cadena de formación y de capacitación.

\section{RefERENCIAIS}

ALTAREJOS, F. et al. Retos educativos de la globalización. Pamplona: EUNSA, 2003

ALTBACH, P. Globalisation and the university: myths and realities in an unequal world. Tertiary Education and management 10 3-25. Netherlands: Kluwer Academic Publishers, 2004 
BARNETT, R. Los límites de la competencia. El conocimiento, la educación superior y la sociedad. Barcelona: Gedisa, 2001

BERNSTEIN, B. Pedagogy, Symbolic Control and Identity. London, Taylor Francis, 1996

CORBELLA, M. La Universidad y el Mercado del aprendizaje claves para comprender el concepto de competencia. Madrid: Dykinson, 2007.

DÍAZ BARRIGA, A. Flexibilización curricular y formación profesional. In: CONGRESO NACIONAL DE INVESTIGACIÓN EDUCATIVA, 2000, México). Conferencias magistrales. México: Consejo Mexicano de Investigación Educativa y Universidad de Colima, 2000.

DUDERSTADT, J. The future of university in an age of knowledge. Journal of Asyncronous learning networks. In SALINAS, J. (1999): Rol del profesor universitario ante los cambios en la era digital. Disponible en http:// www.uib.es/depart/gte/rol.html

KARSETH, B. Currículum restructuring in Higher Education after the Bologna Process: a new Pedagogic Regime?. In Revista Española de Educación Comparada, № 12. Madrid: UNED, 2006

GONZALEZ y WAGENAAR, R. Tuning Educational Structures in Europe. Informe Final. Fase 1. Bilbao: Universidad de Deusto, 2003.

HARRIS, S. Internationalising the university?.In Educational Philosophy and Theory. Volumen 40. Issue 2, 2006

LARA ROS, S. Las competencias básicas necesarias para los ciudadanos del siglo XXI: una revisión de los principales estudios internacionales. ACTAS DEL SIGLO XIV CONGRESO NACIONAL Y III IBEROAMERICANO DE PEDAGOGÍA. Zaragoza: Sociedad Española de Pedagogía, 2008

LYONS, N. (Comp.). El uso de los Portafolios. Propuestas para un nuevo profesionalismo docente. Nueva York: Teachers College Press Columbia University, 1999.

MALAGÓN, L.A. El currículo: dispositivo pedagógico para la vinculación universidad-sociedad". En: Revista Electrónica de la Red de investigación educativa. (en línea). Vol, 1 (Julio-Diciembre de 2004). Disponible en Internet http://revistaiered.org, 2004.

MEYER J.W. et al World Society and the Nation- State. In American Journal of Sociology, 103, 1 pp. 144-.181, 1997

ORTEGA, C. La Formación Continua: Perfil y Competencias del formador. Madrid: UNED, 2008. 
PASTOR, G. Manual de prácticas universitarias de calidad. Herramientas de gestión y seguimiento. Madrid: Netbiblo, 2008.

PEREZ, J.C. La orientación profesional y la evaluación desde el enfoque de competencias. In Revista mexicana de orientación educativa, № 8, marzo-junio, 2006.

RAMOS, G. Internacionalización de la Educación Superior. 1er Encuentro Docente. Educación Superior, Retos y Prospectiva. Universidad del Valle de México, 2003.

RYE, E. Y TORBJORRSEN, A. (2004): Competence-based curricula. The Norwegian (www.cidree.org).

VERGARA CIORDIA, J. Historia del currículo. Madrid, Cuadernos de la UNED, 2006. 